\title{
THE ATOMIC DECOMPOSITION OF HARMONIC FUNCTIONS SATISFYING CERTAIN CONDITIONS OF INTEGRABILITY
}

\section{KRZYSZTOF BOGDAN}

Institute of Mathematics

Technical University of Wroclaw

Wybrzeże Wyspiańskiego 27

50-370 Wrocław, Poland

(Received September 28, 1992 and in revised form May 3, 1993)

\begin{abstract}
Distributions on Euclidean spaces with derivatives of their Poisson integral satisfying certain natural conditions of integrability are represented as sums of weighted atoms. The atomic decomposition is obtained by means of the Calderón reproducing formula.
\end{abstract}

KEY WORDS AND PHRASES. Atomic decomposition, Calderón reproducing formula, harmonic functions, admissible weights.

1980 AMS SUBJECT CLASSIFICATION CODE. 42B99.

\section{INTRODUCTION.}

In [1] Bloom and DeSouza introduced the weighted special atom spaces $\mathbf{B}(\omega)=\left\{f \in D^{\prime}(\mathbf{T})\right.$ : $\left.f=\sum_{n=1}^{\infty} c_{n} b_{n}, \sum_{n=1}^{\infty}\left|c_{n}\right|<\infty\right\}$, where $\mathbf{T}$ is the unit circle in the plane and $D^{\prime}(\mathbf{T})$ are distributions on $\mathbf{T}$. The weight function $\omega$ is a member of a class defined by Bloom and DeSouza in [1]. Each $b_{n}$ is a special atom, that is either $b_{n}(t) \equiv(2 \pi)^{-1}$ or $b_{n}(t)=\omega(|I|)^{-1}|I|^{-1}\left\{\chi_{R}(t)-\chi_{L}(t)\right\}, t \in \mathbf{T}$, where $I$ is an interval in $\mathbf{T}$, with the left and right halves $L$ and $R$. (Our notation is slightly different from that of [1].) As usual $|I|$ denotes the length of $I$ and $\chi_{E}$ the indicator function of $E$. With the usual atomic norm, $\mathbf{B}(\omega)$ becomes a Banach space and yields an atomic characterisation of some well-known spaces on the unit disc. For example, given $\alpha \in(-1,1)$ and $\omega(t)=t^{\alpha}, \mathbf{B}(\omega)$ consists of the real parts of the boundary values (in the sense of distribution) of all those analitic functions $F$, for which $\int_{0}^{1} \int_{0}^{2 \pi}\left|F^{\prime}\left(\mathrm{re}^{\imath \theta}\right)\right| \omega(1-r) d \theta d r<\infty$; see Bloom and DeSouza [1], DeSouza and Sampson [2], DeSouza [3].

The purpose of this paper is to investigate analogous spaces on the real line and, more generally, on the Euclidean spaces of arbitrary dimension. We develop a technique based on the Calderón reproducing formula to prove decomposition theorems in these cases. Also, we obtain a sufficient and necessary condition for the weight function $\omega$ to admit the atomic decomposition of the space $S^{\omega}$ defined below. The method of decomposition is different from that of Bloom and DeSouza [1], and we neither have to investigate the dual space nor the boundary values of elements of $\mathbf{S}^{\omega}$.

The decomposition of $\mathbf{S}^{\omega}$ in terms of the special atoms falls into three steps. The first consists in the decomposition of $\mathbf{S}^{\omega}$ into so called Poisson atoms, which are relevant to properties of $\mathbf{S}^{\omega}$. Then 
we inquire into a class of the Fourier multipliers on $\mathbf{S}^{\omega}$. These are finally used in the proof of the decomposition theorem for the special atoms. In the last part of the paper we sketch out some possible generalisations of the method.

\section{PRELIMINARIES.}

All the vector spaces considered in this paper are over the field of the real numbers. We denote by $\mathbf{N}$ the set of all the non-negative integers and by $\mathbf{N}_{+}$, the set of all the positive integers.

DEFINITION 2.1. Let $(\mathbf{X},\|\cdot\|)$ be a Banach space and let $K \neq \emptyset$ be a bounded subset of $\mathbf{X}$. The set of all $b \in X$, which are of the form

$$
b=\sum_{n=1}^{\infty} c_{n} b_{n}, \sum_{n=1}^{\infty}\left|c_{n}\right|<\infty, b_{n} \in K, n \in \mathbf{N}_{+},
$$

is the atomic space $\mathbf{B}=\mathbf{B}(K)$ spanned by $K$.

We endow the space with the atomic norm $\|b\|_{\mathbf{B}}=\inf \sum_{n=1}^{\infty}\left|c_{n}\right|$, where the infimum is taken over all the possible representations $(2.1)$ of $b \in \mathbf{B}$. The space $\mathbf{B}=\mathbf{B}(K)$ is Banach with respect to the atomic norm. It is often of interest to know whether the spaces $\mathbf{X}$ and $\mathbf{B}$ are equal, that is whether all the elements of $\mathbf{X}$ are of the form (2.1).

LEMMA 2.1. Define $M=\sup \{\|b\|: b \in K\}$. Let $N$ be a positive constant. Suppose that for every $a \in \mathbf{X}$ and $\epsilon>0$ there is some $b \in \mathbf{B}$ such that $\|a-b\| \leq \epsilon$ and $N\|b\|_{\mathbf{B}} \leq\|a\|$. Then $\mathbf{X}=\mathbf{B}$ and also $N\|\cdot\|_{\mathbf{B}} \leq\|\cdot\| \leq M\|\cdot\|_{\mathbf{B}}$.

The proof of Lemma 2.1 is elementary and we omit it here. Note that it can also be obtained as an easy consequence of Theorem 1 in Bonsall [4].

DEFINITION 2.2. A measurable function $\omega$ defined on $\mathbf{R}_{+}=(0, \infty)$ is called a weight if for any interval $I=(a, b), 0<a<b<\infty$, it satisfies

$$
\inf \{\omega(s): s \in I\}>0 \text {. }
$$

From now on, let $n \in \mathbf{N}_{+}$be the dimension of some fixed Euclidean space $\mathbf{R}^{n}$. For a vector $x=\operatorname{col}\left(x_{1}, \ldots, x_{n}\right) \in \mathbf{R}^{n}$, we write $\|x\|$ to denote its Euclidean norm. Let $\mathbf{R}_{+}^{n+1}=\{(x, t): x \in$ $\left.\mathbf{R}^{n}, t>0\right\}$. Given a weight function $\omega$, consider the family $\mathbf{h}^{\omega}$ of all the real valued functions $U$ harmonic in $\mathbf{R}_{+}^{n+1}$, which satisfy the condition:

$$
\|U\|_{\omega}=\int_{\mathbf{R}_{+}^{n+1}}\left\|\nabla_{x} U(x, t)\right\| \omega(t) d x d t<\infty .
$$

Clearly $\|\cdot\|_{\omega}$ is a seminorm. (We write $\nabla_{x} U$ for the vector $\operatorname{col}\left(\partial_{1} U, \ldots, \partial_{n} U\right)$.)

DEFINITION 2.3. Let $\mathbf{S}^{\omega}$ denote the quotient space $\mathbf{h}^{\omega} /\left\|_{\|}\right\|_{\omega}$.

Elements of $\mathbf{S}^{\omega}$ are classes of functions in $\mathbf{h}^{\omega}$, which differ by a linear function of $t$. Indeed, if $U_{1}, U_{2} \in \mathbf{h}^{\omega}$ and $\left\|U_{1}-U_{2}\right\|_{\omega}=0$, then clearly $\nabla_{x} U_{1}=\nabla_{x} U_{2}$ and $\partial_{t}^{2} U_{1}=\partial_{t}^{2} U_{2}$. Therefore $U_{1}-U_{2}=a+b t$ with constants $\mathrm{a}, \mathrm{b}$.

From the mean value property and (2.2), it follows easily that $\mathbf{S}^{\omega}$ is a Banach space.

For $t>0$ and a function $f$ defined on $\mathbf{R}^{n}$, we write, as usual, $f_{t}$ to denote its dilation, that is 
$f_{t}(x)=t^{-n} f(x / t), x \in \mathbf{R}^{n}$. The dilations have a few simple but important properties:

$$
\begin{aligned}
\left\|f_{t}\right\|_{L^{1}\left(\mathbf{R}^{n}\right)} & =\|f\|_{L^{1}\left(\mathbf{R}^{n}\right)} \\
\left(f_{s}\right)_{t} & =f_{s t} \\
f_{t} * g_{t} & =(f * g)_{t} \\
\widehat{\left(f_{t}\right)}(\xi) & =\hat{f}(t \xi), \xi \in \mathbf{R}^{n}, s, t>0, f, g \in L^{1}\left(\mathbf{R}^{n}\right)
\end{aligned}
$$

As usual, $\hat{f}(\xi)=\int_{\mathbf{R}^{n}} f(x) \exp (-i \xi x) d x, \xi \in \mathbf{R}^{n}$, is the Fourier transform of $\mathrm{f}$. Also, we write $f^{y}$ for the translation of $f: f^{y}(x)=f(x-y), x, y \in \mathbf{R}^{n}$, and $f_{t}^{\omega}$ for the weighted dilation defined by $f_{t}^{\omega}=\omega(t)^{-1} f_{t}, t>0$. Finally, let $f_{t}^{y}=\left(f_{t}\right)^{y}$ and $f_{t}^{\omega, y}=\left(f_{t}^{\omega}\right)^{y}, y \in \mathbf{R}^{n}, t>0$.

\section{PROPERTIES OF $\mathbf{S}^{\omega}$.}

DEFINITION 3.1. A weight $\omega$ is called admissible if

$$
\sup _{s>0} \omega(s)^{-1} s \int_{0}^{\infty}(t+s)^{-2} \omega(t) d t<\infty .
$$

Let $\omega$ be a weight function. The boundedness of the expression $\omega(s)^{-1} s \int_{0}^{\infty}(t+s)^{-2} \omega(t) d t$ is equivalent to that of $I=\omega(s)^{-1} s \int_{0}^{\infty} \min \left(s^{-2}, t^{-2}\right) \omega(t) \mathrm{dt}$. Let us write $I=I_{1}+I_{2}$, where $I_{1}=$ $\int_{0}^{s} \omega(t) d t /(s \omega(s))$ and $I_{2}=\int_{s}^{\infty} \omega(t) t^{-2} d t /\left(s^{-1} \omega(s)\right)$. To show that $I$ is bounded, it is enough to verify that $I_{1}$ and $I_{2}$ have finite limits as $s$ tends to $0^{+}$and $\infty$. In some cases this can be easily done using L'Hospital Rule.

EXAMPLE 3.1. The weights

$$
\begin{gathered}
\omega_{1}(t)= \begin{cases}t^{\alpha} & \text { if } 0<t<1, \\
t^{\beta} & \text { if } 1 \leq t<\infty,\end{cases} \\
\omega_{2}(t)= \begin{cases}t^{\alpha}|\ln (t)|^{\gamma} & \text { if } 0<t<1 / 2, \\
1 & \text { if } 1 / 2 \leq t<2, \\
t^{\beta}|\ln (t)|^{\delta} & \text { if } 2 \leq t<\infty,\end{cases}
\end{gathered}
$$

are admissible for every $-1<\alpha, \beta<1$, and $\gamma, \delta \in \mathbf{R}$. To check this we apply the above remark.

The notion of admissibility arises naturally from the investigation of atomic subspaces of $\mathbf{S}^{\omega}$. We define it separately in order to establish firstly some preliminary properties of $\mathbf{S}^{\omega}$ it induces.

LEMMA 3.1. If $\omega$ is admissible, then

$$
\begin{gathered}
s^{-1} \omega(s)^{-1} \rightarrow 0, \text { as } s \rightarrow \infty \\
s \omega(s)^{-1} \rightarrow 0, \text { as } s \rightarrow 0^{+} .
\end{gathered}
$$

Proof. From (3.1) we get $I=\sup _{s>0} \omega(s)^{-1} s^{-1} \int_{0}^{\infty}(t / s+1)^{-2} \omega(t) d t<\infty$. Since $\int_{0}^{\infty}(t / s+$ $1)^{-2} \omega(t) d t \rightarrow \int_{0}^{\infty} \omega(t) d t>0$ as $s \rightarrow \infty$, it follows that $\limsup _{s \rightarrow \infty} \omega(s)^{-1} s^{-1}<\infty$, hence there are positive constants $\delta$ and $c$ such that $\omega(s) \geq c s^{-1}$ for $s>\delta$. We have $\infty>I \geq$ $\sup _{s>0} \omega(s)^{-1} s^{-1} c \int_{\delta}^{\infty}(t / s+1)^{-2} t^{-1} d t$. The integral $\int_{\delta}^{\infty}(t / s+1)^{-2} t^{-1} d t$ tends to $\infty$, so $\omega(s)^{-1} s^{-1}$ tends to 0 , as $s \rightarrow \infty$. Similar reasoning proves (3.3).

For $s>0$ and a function $H$ defined on $\mathbf{R}_{+}^{n+1}$, denote by $H_{s}$ the function given by $H_{s}(x)=$ 
$H(x, s), x \in \mathbf{R}^{n}$. We should not confuse this with the notion of dilation, which is applied to a different class of functions. The functions $H$ and $H_{s}$ may take their values in $\mathbf{R}$ as well as in $\mathbf{R}^{k}, k \in \mathbf{N}_{+}$, depending on the context. In the latter case we call $H=\operatorname{col}\left(H_{1}, \ldots, H_{k}\right)$ harmonic, if $H_{\mathbf{l}}$ is harmonic for each $i=1, \ldots, k$.

As usual, we write $P_{t}$, to denote the Poisson kernel, that is

$$
P_{t}(x)=C_{n} t\left(\|x\|^{2}+t^{2}\right)^{-(n+1) / 2}, x \in \mathbf{R}^{n}, t>0, C_{n}=\Gamma\left(\frac{n+1}{2}\right) \pi^{-(n+1) / 2} .
$$

Note that definition of $P_{t}$ agrees with the notation of the dilation $(\mathrm{P})_{t}$, if we put $P=P_{1}$. Let us recall that elements of $\mathbf{S}^{\omega}$ are classes of functions, which differ by a linear function of $t$.

DEFINITION 3.2. An element $F$ in $\mathbf{S}^{\omega}$ is called regular if for every $s>0$ and $t>0$, the vector valued function $H=\nabla_{x} F$ satisfies the condition

$$
H_{s} \in L^{1}\left(\mathbf{R}^{n}\right) \text { and } H_{t+s}=H_{s} * P_{t}
$$

THEOREM 3.1. Assume that $\omega$ is admissible. Then every $F \in \mathbf{S}^{\omega}$ is regular. Moreover, for every $s>0$, the linear map $\mathbf{S}^{\omega} \ni F \rightarrow \nabla_{x} F_{s} \in L^{1}\left(\mathbf{R}^{n}\right)$ is continuous. In each class $F \in \mathbf{S}^{\omega}$ there is a unique member $U$ such that

$$
\left\|U_{s}\right\|_{L^{\infty}\left(\mathbf{R}^{n}\right)} \rightarrow 0 \text { as } s \rightarrow \infty .
$$

The Poisson integrals of $L^{\infty}\left(\mathbf{R}^{n}\right)$ functions are dense in $\mathbf{S}^{\omega}$.

Proof. For $Y \in \mathbf{R}^{n+1}, r>0$, let $B(Y, r)$ denote the ball in $\mathbf{R}^{n+1}$, which has the radius $r$ and is centered at $Y$. Fix $F \in \mathbf{S}^{\omega}$. Let $y \in \mathbf{R}^{n}, s>0, I=<s / 2,3 s / 2>, c_{I}=\inf _{t \in I} \omega(t)$, and let $B$ stand for $B((y, s), s / 2)$. By the mean value property of $H=\nabla_{x} F$, we get $\|H(y, s)\|=|B|^{-1}$ $\left\|\int_{B} H(x, t) d x d t\right\| \leq c_{I}^{-1}|B|^{-1} \int_{B}\|H(x, t)\| \omega(t) d x d t \leq c_{I}^{-1}|B|^{-1}\|F\|_{\omega}=V^{-1}\left(c_{I}^{-1} s^{-1}\right) s^{-n}\|F\|_{\omega}$, $V$ being the volume of the unit ball in $\mathbf{R}^{n+1}$. By (3.2), the harmonic function $H$ is bounded on $\left\{(x, t): t \geq s, x \in \mathbf{R}^{n}\right\}$ hence $H_{s} \in L^{\infty}\left(\mathbf{R}^{n}\right)$ and $H_{t+s}=H_{s} * P_{t}, t>0$. Now, let $0 \leq t \leq s$. The Young inequality yields $\left\|H_{t}\right\|_{L^{1}\left(\mathbf{R}^{n}\right)} \geq\left\|H_{s}\right\|_{L^{1}\left(\mathbf{R}^{n}\right)}$, conseqently $\|F\|_{\omega}=\int_{0}^{\infty}\left\|H_{t}\right\|_{L^{1}\left(\mathbf{R}^{n}\right)}$ $\omega(t) d t \geq \int_{0}^{s}\left\|H_{s}\right\|_{L^{1}\left(\mathbf{R}^{n}\right)} \omega(t) d t=\left\|H_{s}\right\|_{L^{1}\left(\mathbf{R}^{n}\right)} \int_{0}^{s} \omega(t) d t$. Therefore $\left\|H_{s}\right\|_{L^{1}\left(\mathbf{R}^{n}\right)} \leq c_{s}\|F\|_{\omega}$, with $c_{s}=\left(\int_{0}^{s} \omega(t) d t\right)^{-1}<\infty$, and so the first part of the theorem is proved. Furthermore, since $c_{s} \rightarrow 0$, as $s \rightarrow \infty$, we have

$$
\left\|H_{s}\right\|_{L^{1}\left(\mathbf{R}^{n}\right)} \rightarrow 0 \text { and }\left\|H_{s}\right\|_{L^{\infty}\left(\mathbf{R}^{n}\right)} \rightarrow 0, \text { as } s \rightarrow \infty
$$

Fix some function $T \in F$ and $s>0$. It is not difficult to show that

$$
\sup _{x \in \mathbf{R}^{n}} T_{s}(x)-\inf _{x \in \mathbf{R}^{n}} T_{s}(x) \leq \gamma\left\{\left\|H_{s}\right\|_{L^{1}\left(\mathbf{R}^{n}\right)}+\left\|H_{s}\right\|_{L^{\infty}\left(\mathbf{R}^{n}\right)}\right\},
$$

where $\gamma$ depends only on $\mathrm{n}$. This yields that $T_{s}$ is bounded. The Poisson integral $T_{s} * P_{t-s}(x), t>$ $s, x \in \mathbf{R}^{n}$, is equal to $T$ in $\mathbf{h}^{\omega}$ as a function of $x$ and $t$ (it can be easily defined for $0<t \leq s$ ). Therefore assume that $T$ itself satisfies $T_{t}=T_{s} * P_{t-s}, t>s$, and $T_{s} \in L^{\infty}\left(\mathbf{R}^{n}\right)$. By the assumption and by (3.6) and (3.7), it follows that there is a constant $C$ such that $T_{t}(x) \rightarrow C$ uniformly in $x \in \mathbf{R}^{n}$, as $t \rightarrow \infty$. The function $U=T-C$ satisfies (3.5). Clearly, this condition determines $U$ 
uniquely. Finally, given $\eta>0$, define $\tau_{\eta} F(x, t)=F(x, t+\eta), x \in \mathbf{R}^{n}, t>0$. It is easy to check that $\tau_{\eta} F \rightarrow F$ in $\mathbf{S}^{\omega}$ as $\eta \rightarrow 0^{+}$. Note that $\tau_{\eta} F$ may be represented as the Poisson integral of $U_{\eta} \in L^{\infty}\left(\mathbf{R}^{n}\right)$, with $U \in F$ defined above.

COROLLARY 3.1. If $\omega$ is admissible, $s>0$ and $\xi \in \mathbf{R}^{n}$, then the linear map $\mathbf{S}^{\omega} \ni F \rightarrow$ $\widehat{\nabla}_{x} F_{s}(\xi) \in \mathbf{R}^{n}$ is bounded. Such maps separate elements of $\mathbf{S}^{\omega}$ that is if $E, F \in \mathbf{S}^{\omega}$ and $\widehat{\nabla}_{x} E_{s}(\xi)=$ $\widehat{\nabla}_{x} F_{s}(\xi)$ for every $\xi$ and $s$ then $E=F$.

COROLLARY 3.2. If $\omega$ is admissible, then $\mathbf{S}^{\omega}$ may be identified with the Banach space of all the functions $U \in \mathbf{h}^{\omega}$, which satisfy (3.5).

We shall regard Corollary 3.2 as an alternative definition of $\mathbf{S}^{\omega}$.

\section{THE ATOMIC DECOMPOSITION OF $\mathbf{S}^{\omega}$ IN TERMS OF THE POISSON ATOMS}

We adopt the following notation:

$$
\begin{aligned}
G_{\imath}(x)= & \partial_{\imath} P_{1}(x)=-C_{n}(n+1) x_{\imath}\left(\|x\|^{2}+1\right)^{-(n+3) / 2}, x \in \mathbf{R}^{n}, 1 \leq i \leq n, \\
G= & \operatorname{col}\left(G_{1}, \ldots, G_{n}\right) \\
K_{\imath}(x)= & \nabla G_{\imath}(x)=C_{n}(n+1)(n+3)\left(\|x\|^{2}+1\right)^{-(n+5) / 2} \operatorname{col}\left(x_{1} x_{\imath}, \ldots, x_{n} x_{\imath}\right) \\
& -C_{n}(n+1)\left(\|x\|^{2}+1\right)^{-(n+3) / 2} e_{\imath}, x \in \mathbf{R}^{n}, 1 \leq i \leq n,
\end{aligned}
$$

where $\left\{e_{\imath}: 1 \leq i \leq n\right\}$ is the standard basis in $\mathbf{R}^{n}$.

DEFINITION 4.1. We call Poisson $\omega$-atoms the Poisson integrals $\tilde{G}_{i, s}^{\omega, y}$ of the weighted dilations of the functions $G_{\imath}$, that is

$$
\tilde{G}_{i, s}^{\omega, y}(x, t)=G_{\imath, s}^{\omega, y} * P_{t}(x)=\left(G_{i}\right)_{s}^{\omega, y} * P_{t}(x), x, y \in \mathbf{R}^{n}, s, t>0, i=1, \ldots, n .
$$

Accordingly, let $\tilde{G}_{\imath, s}^{y}=G_{\imath, s}^{y} * P_{t}$.

LEMMA 4.1. There is a constant $C>0$ such that

$$
\left\|\tilde{G}_{1, s}^{0}\right\|_{\omega}=C s \int_{0}^{\infty}(t+s)^{-2} \omega(t) d t, s>0 .
$$

The set of the Poisson $\omega$-atoms is bounded in $\mathbf{S}^{\omega}$ if and only if $\omega$ is admissible.

Proof. It suffices to prove (4.1), the rest being immediate. Let $\mathcal{G}=G_{1}, \mathcal{K}=K_{1}$. Note that the function $t^{-1} \mathcal{G}_{t}(x)=\partial_{1} P_{t}(x)$ is harmonic in $\mathbf{R}_{+}^{n+1}$. It follows easily, that for every $s>0$, the function $\tilde{\mathcal{G}}_{s}(x, t)=s(t+s)^{-1} \mathcal{G}_{t+s}(x), x \in \mathbf{R}^{n}, t>0$, is equal to the Poisson integral of $\mathcal{G}_{s}$. Direct differentiation gives $\nabla_{x} \tilde{\mathcal{G}}_{s}(x, t)=s(t+s)^{-2} \mathcal{K}_{t+s}(x), x \in \mathbf{R}^{n}, s, t>0$. Therefore $\left\|\tilde{\mathcal{G}}_{s}\right\|_{\omega}=s \int_{0}^{\infty}(t+s)^{-2} \omega(t) d t \int_{\mathbf{R}^{n}}\left\|\mathcal{K}_{t+s}(x)\right\| d x$, so (4.1) holds with $0<C=\|\mathcal{K}\|_{L^{1}\left(\mathbf{R}^{n}\right)}<\infty$.

DEFINITION 4.2. Let $\omega$ be admissible. Denote by $\mathbf{G}^{\omega}$ the atomic space spanned in $\mathbf{S}^{\omega}$ by the Poisson $\omega$-atoms.

To state our next result we need some preliminaries.

Assume that $\omega$ is admissible. Let $F \in \mathbf{S}^{\omega}$. Fix $i, 1 \leq i \leq n$, and consider the function $\mathbf{R}_{+}^{n+1} \rightarrow \mathbf{S}^{\omega}$ 
given by $(y, s) \mapsto \dot{G}_{l, s}^{y} \partial_{\imath} F(y, s)$. We claim that it is continuous. Indeed, it is enough to check that the map $(y, s) \mapsto \dot{C}_{i, s}^{y}$ is continuous. Fix $\sigma>0, v \in \mathbf{R}^{n}$. Let $s \rightarrow \sigma$ and $y \rightarrow \vartheta$. We have

$$
\begin{aligned}
\left\|\tilde{G}_{\imath, \sigma}^{\vartheta}-\tilde{G}_{\imath, s}^{y}\right\|_{\omega} & =\int_{\mathbf{R}_{+}^{n+1}}\left\|\nabla_{x} \dot{G}_{\imath, \sigma}^{\vartheta}(x, t)-\nabla_{x} \tilde{G}_{i, s}^{y}(x, t)\right\| \omega(t) d x d t \\
& =\int_{\mathbf{R}_{+}^{n+1}}\left\|\sigma(t+\sigma)^{-2} K_{\imath, t+\sigma}^{\vartheta}(x)-s(t+s)^{-2} K_{\imath, t+s}^{y}(x)\right\| \omega(t) d x d t \\
& =\int_{0}^{\infty}\left\|\sigma(t+\sigma)^{-2} K_{\imath, t+\sigma}^{\vartheta}-s(t+s)^{-2} K_{\imath, t+s}^{y}\right\|_{L^{1}(\mathbf{R})} \omega(t) d t \rightarrow 0
\end{aligned}
$$

by the bounded convergence theorem and by the admissibility of $\omega$. Also, note that

$$
\int_{\mathbf{R}_{+}^{n+1}}\left\|\tilde{G}_{\imath, s}^{y} \partial_{\imath} F(y, s)\right\|_{\omega} d y d s=\int_{\mathbf{R}_{+}^{n+1}}\left\|\tilde{G}_{\imath, s}^{\omega, y}\right\|_{\omega}\left|\partial_{\imath} F(y, s)\right| d y d s \leq C\|F\|_{\omega},
$$

where $C<\infty$ is an upper bound for the $\mathbf{S}^{\omega}$ norms of the Poisson $\omega$-atoms. Therefore the Riemann integral $\int_{\mathbf{R}_{+}^{n+1}} \tilde{G}_{\imath, s}^{y} \partial_{\imath} F(y, s) d y d s$ is well-defined, and so is the following sum

$$
\sum_{i=1}^{n} \int_{\mathbf{R}_{+}^{n+1}} \tilde{G}_{\imath, s}^{y} \partial_{\imath} F(y, s) d y d s, F \in \mathbf{S}^{\omega} .
$$

For $a, b \in \mathbf{R}^{n}, a=\operatorname{col}\left(a_{1}, \ldots, a_{n}\right), b=\operatorname{col}\left(b_{1}, \ldots, b_{n}\right)$, we have $a^{T} b=\sum_{\imath=1}^{n} a_{\imath} b_{\imath}, a^{T}$ being the transpose of a. Therefore we can formally write (4.3) as

$$
\int_{\mathbf{R}_{+}^{n+1}} \tilde{G}_{s}^{y^{T}} \nabla_{y} F(y, s) d y d s, F \in \mathbf{S}^{\omega}
$$

to abbreviate the notation. (We write $\nabla_{y} U$ for $\operatorname{col}\left(\partial_{1} U, \ldots, \partial_{n} U\right)$.)

Let $A=\left(a_{\imath \jmath}: 1 \leq i \leq k, 1 \leq j \leq l\right), B=\left(b_{\imath \jmath}: 1 \leq i \leq l, 1 \leq j \leq m\right)$ be matrices, whose elements are $L^{1}\left(\mathbf{R}^{n}\right)$ functions. Let $A * B=\left(c_{\imath \jmath}: 1 \leq i \leq k, 1 \leq j \leq m\right)$, where $c_{\imath \jmath}=\sum_{r=1}^{l} a_{\imath r} * b_{r j} \epsilon$ $L^{1}\left(\mathbf{R}^{n}\right), 1 \leq i \leq k, 1 \leq j \leq \mathrm{m}$. From the well-known property of the convolution: $\widehat{f * g}(\xi)=$ $\hat{f}(\xi) \hat{g}(\xi), \xi \in \mathbf{R}^{n}$, it follows easily that

$$
\widehat{A * B}(\xi)=\hat{A}(\xi) \hat{B}(\xi), \xi \in \mathbf{R}^{n}
$$

with the natural definition of the Fourier transform of matrices.

THEOREM 4.1. Let $\omega$ be admissible. For every $F \in \mathbf{S}^{\omega}$ the following equality holds

$$
F=-4 \int_{\mathbf{R}_{+}^{n+1}} \tilde{G}_{s}^{y^{T}} \nabla_{y} F(y, s) d y d s \quad \text { (Calderón formula). }
$$

The spaces $\mathbf{S}^{\omega}$ and $\mathbf{G}^{\omega}$ are equal and their norms are equivalent.

Proof. The integral in (4.5) is understood like (4.3) and (4.3'). By Corollary 3.1, it suffices to prove that for every $t>0$ and $\xi \in \mathbf{R}^{n}$, the map $F \mapsto \widehat{\nabla_{x} F_{t}}(\xi)$ takes the same values on both sides of (4.5). Clearly, it is enough to consider the case $\xi \neq 0$. Fix $F \in \mathbf{S}^{\omega}, \xi \in \mathbf{R}^{n} \backslash\{0\}, t>0$. Let

$$
R=\nabla_{x}\left(-4 \int_{\mathbf{R}_{+}^{n+1}} \tilde{G}_{s}^{y^{T}} \nabla_{y} F(y, s) d y d s\right)_{t}(\xi), \xi \neq 0 .
$$


The Riemann integral commutes with continuous linear operators, hence the same result is obtained when the map acts on the integrand. Since $\nabla_{x} \tilde{G}_{s}^{y^{T}}(x, t)=t^{-1} G_{t} * G_{s}^{y^{T}}(x), x, y \in \mathbf{R}^{n}, s, t>0$, we have

$$
R=-4 \int_{\mathbf{R}_{+}^{n+1}}\left(t^{-1} G_{t} * G_{s}^{1 y^{T}}\right) \gamma(\xi) \nabla_{y} F(y, s) d y d s .
$$

Suppose that $F=\int * P_{t}$ for some bounded $\mathrm{f}$. Note that $\nabla_{y} F(y, s)=s^{-1} f * G_{s}(y), s>0, y \in \mathbf{R}^{n}$. Therefore, using (4.4), we get

$$
\begin{aligned}
& R=-4 \int_{\mathbf{R}_{+}^{n+1}}\left(t^{-1} G_{t} * G_{s}^{y^{T}}\right) \mathcal{\Upsilon}(\xi) s^{-1} f * G_{s}(y) d y d s \\
& =-4 \int_{\mathbf{R}_{+}^{n+1}} t^{-1} \widehat{G_{t}}(\xi){\widehat{G_{s}}}^{T}(\xi) \exp (-i y \xi) s^{-1} f * G_{s}(y) d y d s \\
& =-4 \int_{0}^{\infty} t^{-1}{\widehat{G_{t}}}_{(\xi)}{\widehat{G_{s}}}^{T}(\xi) s^{-1} \widehat{F}_{*}(\xi) d s \\
& =-4 \int_{0}^{\infty} t^{-1}\left(G_{t} * G_{s}^{T} * f * G_{s}\right)(\xi) s^{-1} d s \\
& =-4 \int_{0}^{\infty} t^{-1}\left(f * G_{t} * G_{s}^{T} * G_{s}\right) \mathcal{Y}(\xi) s^{-1} d s \\
& =t^{-1} \widehat{f * G}_{t}(\xi)\left(-4 \int_{0}^{\infty}{\widehat{G_{s}}}^{T}(\xi) \widehat{G_{s}}(\xi) s^{-1} d s\right) \\
& =\widehat{\nabla}_{x} F_{t}(\xi)\left(-4 \int_{0}^{\infty} \hat{G}^{T}(s \xi) \hat{G}(s \xi) s^{-1} d s\right) \text {. }
\end{aligned}
$$

To compute $I=\int_{0}^{\infty} \hat{G}^{T}(s \xi) \hat{G}(s \xi) s^{-1} d s$, we make the change of variable $u=\|\xi\| s$, and use the equalities $\widehat{\partial_{\jmath} f}(\zeta)=i \zeta_{\jmath} \hat{f}(\zeta), f, \partial_{\jmath} f \in L^{1}\left(\mathbf{R}^{n}\right)$ and $\widehat{P_{1}}(\zeta)=\exp (-\|\zeta\|), \quad \zeta \in \mathbf{R}^{n}$. We get $I=$ $\int_{0}^{\infty} \hat{G}^{T}(u \xi /\|\xi\|) \hat{G}(u \xi /\|\xi\|) u^{-1} d u=-\int_{0}^{\infty} \exp (-2 u) u d u=-1 / 4$, which proves (4.5), if $F=f * P_{t}$ for some $f \in L^{\infty}\left(\mathbf{R}^{n}\right)$. By (4.2), the linear map $\mathbf{S}^{\omega} \ni F \mapsto-4 \int_{\mathbf{R}_{+}^{n+1}} \tilde{G}_{s}^{y^{T}} \nabla_{y} F(y, s) \omega(s) d y d s \in \mathbf{S}^{\omega}$ is continuous with norm not greater than $4 C n, C$ being as in (4.2). Poisson integrals of $L^{\infty}\left(\mathbf{R}^{n}\right)$ functions are dense in $\mathbf{S}^{\omega}$, hence the map is the identity on the whole of $\mathbf{S}^{\omega}$ and so (4.5) follows. Next, assume that $\omega$ is continuous. For $F \in \mathbf{S}^{\omega}$ and $i=1, \ldots, n$, we clearly have

$$
-4 \int_{\mathbf{R}_{+}^{n+1}} \tilde{G}_{\imath, s}^{y} \partial_{\imath} F(y, s) d y d s=-4 \int_{\mathbf{R}_{+}^{n+1}} \tilde{G}_{\imath, s}^{\omega, y} \partial_{\imath} F(y, s) \omega(s) d y d s .
$$

The second expression is to be understood as a Riemann integral with respect to the finite measure given by the density $\partial_{2} F(y, s) \omega(s)$. The integrand $\tilde{G}_{i, s}^{\omega, y}$ is bounded and continuous as a function with values in $\mathbf{S}^{\omega}$. Therefore the integral is well-defined and may be approximated in $\mathbf{S}^{\omega}$ norm by the Riemann sums $S_{\imath}=-4 \sum_{\jmath=1}^{k} G^{\jmath} \int_{B}, \partial_{\imath} F(y, s) \omega(s) d y d s$, where each $G^{\jmath}$ is a Poisson $\omega$-atom, $E$, are disjoint subsets of $\mathbf{R}_{+}^{n+1}$, and $k \in \mathbf{N}_{+}$. Since clearly $\sum_{\imath=1}^{n}\left\|S_{\imath}\right\|_{\mathbf{G}^{\omega}} \leq 4 \sqrt{n}\|F\|_{\omega}$, the conditions of Lemma 2.1 are fulfilled with $N=1 /(4 \sqrt{n})$ and $M=\sup \left\{\left\|\tilde{G}_{i, s}^{\omega, y}\right\|_{\omega}: y \in \mathbf{R}^{n}, s>0, i=1, \ldots, n\right\}$. In consequence we obtain $\mathbf{S}^{\omega}=\mathbf{G}^{\omega}$ and $\frac{1}{4 \sqrt{n}}\|\cdot\|_{\mathbf{G}^{\omega}} \leq\|\cdot\|_{\omega} \leq M\|\cdot\|_{\mathbf{G}^{\omega}}$. For a discontinuous $\omega$, each term in the expression $-4 \sum_{t=1}^{n} \int_{\mathbf{R}_{+}^{n+1}} \tilde{G}_{i, s}^{\omega, y} \partial_{\imath} F(y, s) \omega(s) d y d s$, may only be understood as a Bochner integral. The integrals are then well-defined because the functions $\mathbf{R}_{+}^{n+1} \rightarrow \mathbf{S}^{\omega}$ given by $(y, s) \mapsto \tilde{G}_{i, s}^{\omega, y}$ are bounded and strongly measurable and the corresponding measures are finite (see Yosida [5] for details on the Bochner integral). The integrals may be approximated in norm by integrals of simple functions all of whose non-zero values are Poisson $\omega$-atoms. Again by Lemma 2.1 , this yields that $\mathbf{S}^{\omega}=\mathbf{G}^{\omega}$ and $\frac{1}{4 \sqrt{n}}\|\cdot\|_{\mathbf{G}^{\omega}} \leq\|\cdot\|_{\omega} \leq M\|\cdot\|_{\mathbf{G}^{\omega}}$. 
REMARK 4.1. For an admissible and discontinuous weight $\omega$, define $\omega^{*}(s)=s \int_{0}^{\infty}(t+s)^{-2} \omega(t) d t$, $s>0$. It may be easily verified that the weight $\omega^{*}$ is admissible and continuous and moreover we have $\mathbf{S}^{\omega}=\mathbf{S}^{\boldsymbol{\omega}^{*}}$.

Let us make a digression here to show how the atomic decomposition of the space $\mathbf{S}^{\omega}$ may be expressed in terms of the boundary values of its elements.

For $p \geq 1$ satisfying $n(1-1 / p)<1$, define $\omega(t)=t^{-n(1-1 / p)}, t>0$. Note that

$$
\begin{aligned}
\left\|G_{i, s}^{w, y}\right\|_{L^{p}\left(\mathbf{R}^{n}\right)}^{p} & =\int_{\mathbf{R}^{n}}\left|s^{n(1-1 / p)} s^{-n} G_{\imath}((x-y) / s)\right|^{p} d x \\
& =\int_{\mathbf{R}^{n}} s^{-n}\left|G_{\imath}(z)\right|^{p} s^{n} d z=\left\|G_{1}\right\|_{L^{p}\left(\mathbf{R}^{n}\right)}^{p}<\infty
\end{aligned}
$$

hence by the decomposition of $\mathbf{S}^{\omega}$ into Poisson $\omega$-atoms $\dot{G}_{\imath, s}^{\omega, y}$, the boundary value functions of its elements are all in $L^{p}\left(\mathbf{R}^{n}\right)$.

COROLLARY 4.1. Let $p \geq 1$ satisfy $p<n /(n-1)$. If $\omega(t)=t^{-n(1-1 / p)}, t>0$, then the following equality holds in $L^{p}\left(\mathbf{R}^{n}\right)$ :

$$
F_{0}=-4 \int_{\mathbf{R}_{+}^{n+1}} G_{s}^{y^{T}} \nabla_{y} F(y, s) d y d s, F \in \mathbf{S}^{\omega}, \text { where } F_{0}(x)=F\left(x, 0^{+}\right) \text {a.e. }
$$

REMARK 4.2. Let $\omega$ be an admissible weight function. Consider the class $\mathbf{h}^{\prime \omega}$ of all the real valued functions $U$ harmonic in $\mathbf{R}_{+}^{n+1}$, which satisfy the condition $\|U\|_{\omega}^{\prime}=\int_{\mathbf{R}_{+}^{n+1}}\left|\partial_{t} U(x, t)\right|$ $\omega(t) d x d t<\infty$. Define $\mathbf{S}^{\prime \omega}=\mathbf{h}^{\prime \omega} /\|\cdot\|_{\omega}^{\prime}$. It is easy to check that the Poisson $\omega$-atoms form a bounded set in $\mathbf{S}^{\prime \omega}$. This yields $\mathbf{S}^{\omega} \subseteq \mathbf{S}^{\prime \omega}$. Define the function $M$ by $M(x)=\left.\partial_{t} P_{t}(x)\right|_{t=1}, x \in \mathbf{R}^{n}$. The method described above may be applied to decompose $\mathbf{S}^{\prime \omega}$ into the Poisson integrals of the weighted dilations of $M$. The same functions form a bounded set in $\mathbf{S}^{\omega}$, hence the spaces $\mathbf{S}^{\omega}$ and $\mathbf{S}^{\omega}$ are equal and their norms are equivalent. We omit the details.

REMARK 4.3. The basic references for the Calderón reproducing formula in context of atomic decomposition are Frazier and Yawerth [6] and Frazier et al. [7]. For example an atomic decomposition of the homogeneous Besov spaces $\dot{B}_{p}^{\alpha, q}$ is stated in [7] (see Frazier and Yawerth [8] for the proof). Taking advantage of the result and of Theorem 4.1, it may be verified that the Poisson integrals of the distributions in $\dot{B}_{1}^{\alpha, 1}$, form the space $S^{\omega}$ for $\omega(t)=t^{-\alpha}, \alpha \in(-1,1)$. We note here that the atomic decomposition is understood more generally in Frazier and Yawerth [6] and Frazier et al. [7] than in this paper (see also Feichtinger and Gröchenig [9] for a group theoretical point of view). Our approach is rather in spirit of the decomposition of the space $\dot{B}_{1}^{0,1}$ which is treated separately in [7], however there are changes due to the fact that special atoms defined below are not smooth nor radial and that we consider a wide class of weight functions. Also, recall that we did not assume that the boundary values of elements in $\mathbf{S}^{\omega}$ are distributions. Now this fact may be verified using the atomic decomposition of $\mathbf{S}^{\omega}$ into Poisson $\omega$-atoms (regard (3.2), (3.3) as estimates for the size of atoms). We omit the details since the result will not be exploited in this paper.

\section{FOURIER MULTIPLIERS ON $\mathbf{S}^{\omega}$}

Assume that $\omega$ is admissible. We focus our attention on the Hilbert transform type Fourier 
multipliers $\mathcal{M}: \mathbf{S}^{\omega} \rightarrow \mathbf{S}^{\omega}$ satisfying

$$
\nabla_{x} \widehat{\mathcal{M}} F_{t}(\xi)=m(\xi) \widehat{\nabla_{x} F_{t}}(\xi), \xi \in \mathbf{R}^{n}, t>0, F \in \mathbf{S}^{\omega}
$$

where $m$ is a real or complex function homogeneous of degree 0 .

There are (only) formal reasons for introducing the gradient $\nabla_{x}$ in (5.1) since we did not give a meaning to $\widehat{F}_{t}(\xi)$ and we cannot generally use a more preferable $\widehat{\mathcal{M F}}_{t}(\xi)=m(\xi) \widehat{F}_{t}(\xi)$. Similarly we did not established what the boundary value, say $F_{0}$, of $F \in \mathbf{S}^{\omega}$ is, and we cannot write $\widehat{\mathcal{M F}}_{0}(\xi)=m(\xi) \widehat{F}_{0}(\xi)$ which is in fact the idea behind (5.1). The following theorem states some conditions on the function $m$ sufficient for existence of the operator $\mathcal{M}: \mathbf{S}^{\omega} \rightarrow \mathbf{S}^{\omega}$ satisfying (5.1). Note that by Corollary 3.1 such an operator is unique.

THEOREM 5.1. Let $m$ be homogeneous of degree $0, n$ tımes continuously differentiable on $\mathbf{R}^{n} \backslash\{0\}$. If $m=m_{1}+i m_{2}$, where $m_{1}, m_{2}$ are respectively even and odd real functions, then there is an operator $\mathcal{M}$ satisfying (5.1) which maps $\mathbf{S}^{\omega}$ continuously into $\mathbf{S}^{\omega}$.

In particular Riesz operators $R_{\mathfrak{\imath}}$ given by the functions $m_{\mathfrak{z}}(\xi)=-i \xi_{\imath}\|\xi\|^{-1}, \xi \in \mathbf{R}^{n}, 1 \leq i \leq n$, are bounded on $\mathbf{S}^{\omega}$, which means that it is invariant under generalized conjugation.

First, we prove an auxiliary fact. Let $\epsilon>0$ and $d \in \mathbf{N}$ be fixed. As usual $\mathbf{C}^{d}[-\epsilon, \epsilon]$ denotes the class of all the functions defined on $[-\epsilon, \epsilon]$, which have continuous derivatives up to the $d$-th order. Put $\|f\|_{d}=\sup \left\{\left|f^{(k)}(x)\right|: x \in[-\epsilon, \epsilon], k=0, \ldots, d\right\}, f \in \mathbf{C}^{d}[-\epsilon, \epsilon]$.

LEMMA 5.1. Given $\epsilon>0$ and $d \in \mathbf{N}$, there is a constant $C$ such that for every $f \in \mathbf{C}^{d}[-\epsilon, \epsilon]$ and $|u| \geq 1$, the following inequality holds

$$
\left|\int_{-\epsilon}^{+\epsilon} f(s)(1-i u s)^{-(d+2)} d s\right| \leq C\|f\|_{d}|u|^{-(d+1)} .
$$

Proof. Let us write $C_{d}$ rather than $C$ to distinguish between different numbers $C$ depending on $\mathrm{d}$. Assume that $|u| \geq 1$. The proof is by induction on $d \in \mathbf{N}$. For $d=0$ we have:

$$
\left|\int_{-\epsilon}^{+c} f(s)(1-i u s)^{-2} d s\right| \leq\|f\|_{0} \int_{-\infty}^{\infty}\left(1+(u s)^{2}\right)^{-1} d s=\pi\|f\|_{0}|u|^{-1}
$$

hence $C_{0}=\pi$. Next, integration by parts yields

$$
\begin{aligned}
\left|\int_{-\epsilon}^{+\epsilon} f(s)(1-i u s)^{-(d+3)} d s\right| \leq & \left|-i(d+2)^{-1} u^{-1} f(s)(1-i u s)^{-(d+2)}\right|_{-\epsilon}^{+\epsilon} \\
& +i(d+2)^{-1} u^{-1} \int_{-\epsilon}^{+\epsilon} f^{\prime}(s)(1-i u s)^{-(d+2)} d s \mid \\
\leq & 2(d+2)^{-1}\|f\|_{0}|u|^{-(d+3)} \epsilon^{-(d+2)} \\
& +(d+2)^{-1}|u|^{-1} C_{d}\left\|f^{\prime}\right\|_{d}|u|^{-(d+1)} \\
\leq & C_{d+1}\|f\|_{d+1}|u|^{-(d+2)}
\end{aligned}
$$

where $C_{d+1}=(d+2)^{-1}\left\{2 \epsilon^{-(d+2)}+C_{d}\right\}$.

Proof of Theorem 5.1. By Theorem 4.1 it is sufficient to show that there is an operator $\mathcal{M}$ with values in $\mathbf{S}^{\omega}$ satisfying (5.1) and bounded on the set of all the Poisson $\omega$-atoms; a typical application of atomic decomposition. Such an operator extends uniquely to a continuous map from $\mathbf{S}^{\omega}$ into 
itself. From the Plancherel theorem there is a function $g \in L^{2}\left(\mathbf{R}^{n}\right)$ satisfying $\hat{g}(\xi)=m(\xi) \exp (-\|\xi\|)$ a.e. In fact, $g$ may be uniquely chosen to be smooth. Given $\imath, j: 1 \leq i, j \leq n$, consider $f=\partial_{\imath} \partial_{\text {, g }}$. We claim that $f$ is real and integrable. Let $w(\xi)=-\xi_{2} \xi_{3} m(\xi), \xi=\operatorname{col}\left(\xi_{1}, \ldots, \xi_{n}\right) \in \mathbf{R}^{n}$. Fix $x \in \mathbf{R}^{n}$. By the Fourier inversion formula we have

$$
f(x)=c_{n} \int_{\mathbf{R}^{n}} w(\xi) \exp (-\|\xi\|) \exp (i \xi x) d \xi, c_{n}=(2 \pi)^{-n} .
$$

To compute $f(x)$, we introduce polar coordinates $r \in(0, \infty), \sigma \in S^{n-1}, S^{n-1}$ being the unit sphere in $\mathbf{R}^{n}$, and we integrate $(n+1)$ times by parts with respect to $r$. We obtain:

$$
\begin{aligned}
f(x) & =c_{n} \int_{S^{n-1}} w(\sigma) \int_{0}^{\infty} \exp (-r(1-i \sigma x)) r^{n+1} d r d \sigma \\
& =c_{n}(n+1) ! \int_{S^{n-1}} w(\sigma)(1-i \sigma x)^{-(n+1)} \int_{0}^{\infty} \exp (-r(1-\imath \sigma x)) d r d \sigma .
\end{aligned}
$$

Finally, we get:

$$
f(x)=c_{n}(n+1) ! \int_{S^{n-1}} w(\sigma)(1-i \sigma x)^{-(n+2)} d \sigma .
$$

Everything is now easy for $n=1: f(x)=\pi^{-1}\left\{w(-1)(1+i x)^{-3}+w(1)(1-i x)^{-3}\right\}$. Therefore assume that $n \geq 2$. Let $\|x\| \geq 1$. We make the following change of variable:

$$
\left\{\begin{aligned}
\sigma & =\eta \sin \varphi+\tau \cos \varphi \\
d \sigma & =\sin ^{n-2} \varphi d \eta d \varphi, \eta \in S_{x}^{n-2}=\left\{y \in S^{n-1}:(y, x)=0\right\}, \tau=x /\|x\|, \varphi \in[0, \pi) .
\end{aligned}\right.
$$

(We can identify $S_{x}^{n-2}$ with the unit sphere in $\mathbf{R}^{n-1}$, and $d \eta$ with the $(n-2)$-dimensional spherical measure. As usual $(\cdot, \cdot)$ denotes the inner product in $\mathbf{R}^{n}$.) We get:

$$
f(x)=c_{n}(n+1) ! \int_{S_{x}^{n-2}} \int_{0}^{\pi} w(\eta \sin \varphi+\tau \cos \varphi)(1-i\|x\| \cos \varphi)^{-(n+2)} \sin ^{n-2} \varphi d \varphi d \eta .
$$

Another change of variables: $s=\cos \varphi, s \in[-1,1]$, gives

$$
f(x)=c_{n}(n+1) ! \int_{S_{x}^{n-2}} \int_{-1}^{+1} w\left(\eta\left(1-s^{2}\right)^{1 / 2}+\tau s\right)(1-i\|x\| s)^{-(n+2)}\left(1-s^{2}\right)^{(n-3) / 2} d s d \eta .
$$

Fix $\epsilon \in(0,1)$ and use Lemma 5.1 to obtain the estimate

$$
\left|\int_{-\epsilon}^{+\epsilon} w\left\{\eta\left(1-s^{2}\right)^{1 / 2}+\tau s\right\}(1-i\|x\| s)^{-(n+2)}\left(1-s^{2}\right)^{(n-3) / 2} d s\right| \leq C\|x\|^{(n-1)},
$$

where $C<\infty$ depends only on $n, \epsilon$, and the supremum over $S^{n-1}$ of all the partial derivatives of the $n$-th order of $\mathrm{m}$. We see immediately that there is a constant $C^{\prime}<\infty$, such that $|f(x)| \leq$ $C^{\prime}\|x\|^{-(n+1)},\|x\| \geq 1$. Since $f$ is continuous, this yields $f \in L^{1}\left(\mathbf{R}^{n}\right)$.

Let $S_{+}^{n-1}=\left\{\sigma=\left(\sigma_{1}, \ldots, \sigma_{n}\right): \sigma_{1}>0\right\}$. From (5.2), for every $x \in \mathbf{R}^{n}$, we have

$$
f(x)=c_{n}(n+1) ! \int_{S_{+}^{n-1}}\left\{w(\sigma)(1-i \sigma x)^{-(n+2)}+w(-\sigma)(1+i \sigma x)^{-(n+2)}\right\} d \sigma, c_{n}=(2 \pi)^{-n},
$$

the integrand being real by the assumption on $m$. Therefore $f$ is real valued. Note that either in the case $n=1$, or in the case $n \geq 2, g$ is necessarily real valued as well. Let $E_{\imath}=\partial_{\imath} g, 1 \leq i \leq n$. Fix $y \in$ $\mathbf{R}^{n}, s>0$ and $i, 1 \leq i \leq \mathrm{n}$. Let us recall that $\tilde{G}_{i, s}^{\omega, y}(x, t)=\omega(s)^{-1} s(t+s)^{-1} G_{i, t+s}^{y}(x), x \in \mathbf{R}^{n}, t>0$. A simple verification shows that $\mathcal{M}\left(\tilde{G}_{\imath, s}^{\omega, y}\right)(x, t)=\omega(s)^{-1} s(t+s)^{-1} E_{\imath, t+s}^{y}(x), x \in \mathbf{R}^{n}, t>0$ satisfies 
(5.1) for every Poisson $\omega$-atom F. By means of the Fourier transform it is easy to check that $\mathcal{M} \tilde{G}_{t, s}^{\omega, y}$ is a harmonic function on $\mathbf{R}_{+}^{n+1}$. Clearly, it is also rcal valued and vanishes at infinity. Note that

$$
\begin{aligned}
\left\|\mathcal{M} \tilde{G}_{\imath, s}^{n, y}\right\|_{\omega} & =\int_{\mathbf{R}_{+}^{n+1}} \omega(s)^{-1} s(t+s)^{-1}\left\|\nabla_{x} E_{\imath, t+s}^{y}(x)\right\| \omega(t) d x d t \\
& =\int_{0}^{\infty} \omega(s)^{-1} s(t+s)^{-2}\left\|\left(\nabla_{x} E_{\imath}\right)_{t+s}^{y}\right\|_{L^{1}\left(\mathbf{R}^{n}\right)} \omega(t) d t \\
& =\left\|\nabla_{x} E_{\imath}\right\|_{L^{1}\left(\mathbf{R}^{n}\right)} \omega(s)^{-1} s \int_{0}^{\infty}(t+s)^{-2} \omega(t) d t .
\end{aligned}
$$

Since $\nabla_{x} E_{\imath}$ is integrable and $\omega$ is admissible, the set $\left\{\mathcal{M} \tilde{G}_{\imath, s}^{\omega, y}: y \in \mathbf{R}^{n}, s>0,1 \leq i \leq n\right\}$ is bounded in $\mathbf{S}^{\omega}$. This proves the theorem.

\section{DECOMPOSITION OF $\mathbf{S}^{\omega}$ IN TERMS OF SPECIAL ATOMS}

Let us introduce the following functions:

$$
b_{\imath}(x)=-2^{-n} \operatorname{sgn}\left(x_{\imath}\right) \prod_{\jmath=1}^{n} \chi_{(-1,1)}\left(x_{\jmath}\right), x \in \mathbf{R}^{n}, 1 \leq i \leq n .
$$

We are now in a position to prove that $\mathbf{S}^{\omega}$ is equal to the atomic space generated by the functions $b_{\boldsymbol{t}}$. Note that these functions give a natural extension of the notion of the special atom on $\mathbf{T}$.

DEFINITION 6.1. We call special $\omega$-atoms the Poisson integrals $\tilde{b}_{i, s}^{\omega, y}$ of the weighted dilations of the functions $b_{\imath}$, that is

$$
\tilde{b}_{i, s}^{\omega, y}(x, t)=b_{\imath, s}^{\omega, y} * P_{t}(x)=\left(b_{\imath}\right)_{s}^{\omega, y} * P_{t}(x), x, y \in \mathbf{R}^{n}, s, t>0, i=1, \ldots, n .
$$

Accordingly, let $\tilde{b}_{\imath, s}^{y}=b_{\imath, s}^{y} * P_{t}$.

LEMMA 6.1. There are positive constants $C_{1}, C_{2}$ such that

$$
C_{1} s \int_{0}^{\infty}(t+s)^{-2} \omega(t) d t \leq\left\|\tilde{b}_{1, s}^{0}\right\|_{\omega} \leq C_{2} s \int_{0}^{\infty}(t+s)^{-2} \omega(t) d t, s>0 .
$$

The set of the special $\omega$-atoms is bounded in $\mathbf{S}^{\omega}$ if and only if $\omega$ is admissible.

Proof. It suffices to prove (6.1), the rest being immediate. Let $\mathcal{B}=b_{1}, \tilde{\mathcal{B}}_{s}=\tilde{b}_{1, s}^{0}, s>0$. We have

$$
\begin{aligned}
\left\|\tilde{\mathcal{B}}_{s}\right\|_{\omega} & =\int_{\mathbf{R}_{+}^{n+1}}\left\|\nabla_{x} \tilde{\mathcal{B}}_{s}(x, t)\right\| \omega(t) d x d t=\int_{\mathbf{R}_{+}^{n+1}}\left\|t^{-1} \mathcal{B}_{s} * G_{t}(x)\right\| \omega(t) d x d t \\
& =\int_{0}^{\infty} t^{-1}\left\|\mathcal{B}_{s} * G_{t}\right\|_{L^{1}\left(\mathbf{R}^{n}\right)} \omega(t) d t=\int_{0}^{\infty} t^{-1}\left\|\mathcal{B}_{s / t} * G\right\|_{L^{1}\left(\mathbf{R}^{n}\right)} \omega(t) d t .
\end{aligned}
$$

Assume that there are constants $c_{1}>0, c_{2}>0$, such that for every $r>0$,

$$
c_{1} \min \left(r, r^{-1}\right) \leq\left\|\mathcal{B}_{r} * G\right\|_{L^{1}\left(\mathbf{R}^{n}\right)} \leq c_{2} \min \left(r, r^{-1}\right) .
$$

By (6.2) we obtain

$$
\begin{aligned}
\left\|\tilde{\mathcal{B}}_{s}\right\|_{\omega} & =\int_{0}^{\infty} t^{-1}\left\|\mathcal{B}_{s / t} * G\right\|_{L^{1}\left(\mathbf{R}^{n}\right)} \leq c_{2} \int_{0}^{\infty} t^{-1} \min (s / t, t / s) \omega(t) d t \\
& \leq 4 c_{2} s \int_{0}^{\infty}(t+s)^{-2} \omega(t) d t .
\end{aligned}
$$

Likewise $\left\|\tilde{\mathcal{B}}_{s}\right\|_{\omega} \geq c_{1} s \int_{0}^{\infty}(t+s)^{-2} \omega(t) d t$, so (6.1) holds with $C_{1}=c_{1}$ and $C_{2}=4 c_{2}$, if only (6.2) is valid. To prove $(6.2)$, we show first that

$$
\left\|\mathcal{B}_{r} * G\right\|_{L^{1}\left(\mathbf{R}^{n}\right)} \leq c_{2} \min \left(r, r^{-1}\right), r>0 .
$$


Note that $\int_{\mathbf{R}^{n}} \mathcal{B}(x) d x=0$ and that the map $\mathbf{R}^{n} \ni y \mapsto G^{y} \in L^{1}\left(\mathbf{R}^{n}\right)$ is Lipschitz continuous, that is $\left\|G^{y}-G^{\prime}\right\|_{L^{1}\left(\mathbf{R}^{n}\right)} \leq C|y|, y \in \mathbf{R}^{n}$, for a constant C. By Fubini-Tonelli theorem, this yields

$$
\begin{aligned}
\left\|\mathcal{B}_{r} * G\right\|_{L^{1}\left(\mathbf{R}^{n}\right)} & =\int_{\mathbf{R}^{n}}\left\|\int_{\mathbf{R}^{n}} \mathcal{B}_{r}(y) G(x-y) d y\right\| d x=\int_{\mathbf{R}^{n}}\left\|\int_{\mathbf{R}^{n}} \mathcal{B}_{r}(y)\left[G(x-y)-C^{\prime}(x)\right] d y\right\| d x \\
& \leq \int_{\mathbf{R}^{n}} \int_{\mathbf{R}^{n}}\left|\mathcal{B}_{r}(y)\right|\left\|G^{\prime}(x-y)-G(x)\right\| d x d y=\int_{\mathbf{R}^{n}}\left|\mathcal{B}_{r}(y)\right|\left\|G^{y}-G^{\prime}\right\|_{L^{\prime}\left(\mathbf{R}^{n}\right)} d y \\
& \leq C r \int_{\mathbf{R}^{n}}\left|\mathcal{B}_{r}(y) \| y / r\right| d y=c^{\prime} r, r>0, \text { where } c^{\prime}=C \int_{\mathbf{R}^{n}}|\mathcal{B}(y) y| d y .
\end{aligned}
$$

Note also that $\left\|\mathcal{B}_{r} * C^{\prime}\right\|_{L^{1}\left(\mathbf{R}^{n}\right)}=\| \mathcal{B} *\left(i_{1 / r} \|_{L^{\prime}\left(\mathbf{R}^{n}\right)}, r>0\right.$. Changing the roles of $\mathcal{B}$ and $G$ above, we get $\left\|\mathcal{B}_{r} * G^{\prime}\right\|_{L^{\prime}\left(\mathbf{R}^{n}\right)}=\left\|\mathcal{B} * G_{1 / r}\right\|_{L^{1}\left(\mathbf{R}^{n}\right)} \leq c^{\prime \prime} r^{-1}, r>0$, with a constant $c^{\prime \prime}$. Take $c_{2}=\max \left(c^{\prime}, c^{\prime \prime}\right)$ to obtain (6.3). Next, we prove

$$
c_{1} \min \left(r, r^{-1}\right) \leq\left\|\mathcal{B}_{r} * G\right\|_{L^{1}\left(\mathbf{R}^{n}\right)}, r>0
$$

Clearly, it is enough to verify that

$$
c_{1} \min \left(r, r^{-1}\right) \leq\left\|\mathcal{B}_{r} * G_{1}\right\|_{L^{1}\left(\mathbf{R}^{n}\right)}, r>0 .
$$

Define $p(x)=\frac{1}{2}\left[\lambda_{(-1,0)}(x)-\chi_{(0,1)}(x)\right], x \in \mathbf{R}$, and $q(x)=\frac{1}{2} \lambda_{(-1,1)}(x), x \in \mathbf{R}$. We have $\hat{p}(\zeta)=$ $1 / 2\left[\int_{-1}^{0} \exp (-\imath \zeta x) d x-\int_{0}^{1} \exp (-i \zeta x) d x\right]=\imath(1-\cos \zeta) / \zeta, \zeta \in \mathbf{R} \backslash\{0\}, \hat{p}(0)=0$, and, similarly, $\hat{q}(\zeta)=\sin \zeta / \zeta, \zeta \in \mathbf{R} \backslash\{0\}, \hat{q}(0)=1$. Since $\mathcal{B}$ is a tensor product, the same is true for $\hat{\mathcal{B}}$. Explicitly,

$$
\hat{\mathcal{B}}(\xi)=\hat{p}\left(\xi_{1}\right) \prod_{\imath=2}^{n} \hat{q}\left(\xi_{\imath}\right), \xi=\operatorname{col}\left(\xi_{1}, \ldots, \xi_{n}\right) \in \mathbf{R}^{n}
$$

Recall that $\hat{G}_{1}(\xi)=i \xi_{1} \exp (-\|\xi\|), \xi \in \mathbf{R}^{n}$. Fix $\xi \in \mathbf{R}^{n}$ such that $\xi_{1} \neq 0$, and consider the expression

$$
I_{1}=r^{-1} \widehat{\mathcal{B}}_{r} \widehat{G}_{1}(\xi)=r^{-1} \hat{\mathcal{B}}(r \xi) \hat{G}_{1}(\xi)=\left\{i \hat{p}\left(r \xi_{1}\right) /\left(r \xi_{1}\right) \prod_{\imath=2}^{n} \hat{q}\left(r \xi_{\imath}\right)\right\} \xi_{1}^{2} \exp (-\|\xi\|), r>0 .
$$

We see immediately that $I \rightarrow \frac{1}{2} \xi_{1}^{2} \exp (-\|\xi\|)>0$, as $r \rightarrow 0^{+}$. Next, for $r>0, \xi_{1}=\xi_{2}=\ldots=\xi_{n}=$ $\frac{1}{4} \pi r^{-1}$, consider the expression

$$
I_{2}=r \mathcal{B}_{r} * G_{1}(\xi)=\left\{i \hat{p}\left(r \xi_{1}\right)\left(r \xi_{1}\right) \prod_{\imath=2}^{n} \hat{q}\left(r \xi_{\imath}\right)\right\} \exp (-\|\xi\|) .
$$

We check at once that $I_{2} \rightarrow(1-\sqrt{2} / 2)(2 \sqrt{2} / \pi)^{n-1}>0$, as $r \rightarrow \infty$. Since, for $f \in L^{1}\left(\mathbf{R}^{n}\right)$, we have $\|\hat{f}\|_{L^{\infty}\left(\mathbf{R}^{n}\right)} \leq\|f\|_{L^{1}\left(\mathbf{R}^{n}\right)}$, it follows that $\liminf _{r \rightarrow 0^{+}} r^{-1}\left\|\mathcal{B}_{r} * G_{1}\right\|_{L^{1}\left(\mathbf{R}^{n}\right)}>0$ and $\liminf _{r \rightarrow \infty} r \|$ $\mathcal{B}_{r} * G_{1} \|_{L^{1}\left(\mathbf{R}^{n}\right)}>0$. Since $\left\|\mathcal{B}_{r} * G_{1}\right\|_{L^{1}\left(\mathbf{R}^{n}\right)}$ is a positive continuous function of $r>0,(6.5)$ holds with a constant $c_{1}>0$.

DEFINITION 6.2. Assume that $\omega$ is admissible. Let $\mathbf{B}^{\omega}$ denote the atomic space spanned by the special $\omega$-atoms.

To state the next result we need some preliminary steps.

DEFINITION 6.3. Let $\omega$ be admissible. Fix $s>0$ and $i: 1 \leq i \leq n$. For $F \in \mathbf{S}^{\omega}$ let

$$
F_{0} * b_{\imath, s}=-4 \int_{\mathbf{R}_{+}^{n+1}} G_{r}^{y^{T}} * b_{\imath, s} \nabla_{y} F(y, r) d y d r=-4 \sum_{\jmath=1}^{n} \int_{\mathbf{R}_{+}^{n+1}} G_{\jmath, r}^{y} * b_{\imath, s} \partial j F(y, r) d y d r .
$$


Each term in the sum is understood as the Riemann integral of the function $\mathbf{R}_{+}^{n+1} \rightarrow L^{1}\left(\mathbf{R}^{n}\right)$ given by $(y, r) \mapsto C_{j, r}^{y} * b_{\imath, s} \partial_{j} F(y, r)$. The symbol of convolution in $F_{0} * b_{\imath, s}$ may be fully justified because $\lim _{t \rightarrow 0^{+}} F_{t}$ exists as a distribution. However, we do not develope this point here, and the notation $F_{0} * b_{t, s}$ is purely formal. In particular the symbol $F_{0}$ alone has no generally prescribed meaning. To prove that $F_{0} * b_{l, s}$ is well-defined, fix $s>0, \imath$ and $j: 1 \leq i, j \leq n$. Clearly, the map $\mathbf{R}_{+}^{n+1} \ni(y, r) \mapsto$ $G_{\jmath, r}^{y} * b_{\imath, s} \in L^{1}\left(\mathbf{R}^{n}\right)$ is continuous. From (6.2) we have $\left\|G_{\jmath, r}^{y} * b_{\imath, s}\right\|_{L^{1}\left(\mathbf{R}^{n}\right)}=\left\|G_{\jmath} * b_{\imath, s / r}\right\|_{L^{1}(\mathbf{R})}, r>0$. By Lemma 3.1, the expression $\min (s / r, r / s) \omega(r)^{-1}$ is bounded, thus

$$
\begin{aligned}
\int_{\mathbf{R}_{+}^{n+1}}\left\|G_{\jmath, r}^{y} * b_{\imath, s}\right\|_{L^{1}\left(\mathbf{R}^{n}\right)}\left|\partial_{\jmath} F(y, r)\right| d y d r & \leq c_{2} \int_{\mathbf{R}_{+}^{n+1}} \min (s / r, r / s) \omega(r)^{-1}\left|\partial_{\jmath} F(y, r)\right| \omega(r) d y d r \\
& \leq C\|F\|_{\omega} \text { for a constant } C .
\end{aligned}
$$

This justifies the definition. Note that we have actually proved that the linear map $\mathbf{S}^{\omega} \ni F \mapsto$ $F_{0} * b_{\imath, s} \in L^{1}\left(\mathbf{R}^{n}\right)$ is continuous. Suppose that $F$ is a finite sum of Poisson $\omega$-atoms, and let $f=F_{0}$ i.e. $f(x)=F(x, 0)$. By Corollary 4.1 with $p=1$, we have $f=-4 \int_{\mathbf{R}_{+}^{n+1}} G_{r}^{y^{T}} \nabla_{y} F(y, r) d y d r$ in $L^{1}\left(\mathbf{R}^{n}\right)$, for every $s>0$ and $1 \leq i \leq n$. It follows easily that $f * b_{\imath, s}=-4 \int_{\mathbf{R}_{+}^{n+1}} G_{r}^{y^{T}} * b_{\imath, s} \nabla_{y} F(y, r) d y d r$ in $L^{1}\left(\mathbf{R}^{n}\right)$ for every $s>0$ and $1 \leq \imath \leq n$, so, as one could expect, $F_{0} * b_{\imath, s}=f * b_{\imath, s}$. Let us denote by $F_{0} * b_{s}$ the vector $\operatorname{col}\left(F_{0} * b_{1, s}, \ldots, F_{0} * b_{n, s}\right)$.

LEMMA 6.2. Let $\omega$ be admissible. The map $\Phi: \mathbf{S}^{\omega} \rightarrow \mathbf{S}^{\omega}$ given by $F \mapsto \Phi F$, where $\Phi F$ is the Riemann integral

$$
\int_{\mathbf{R}_{+}^{n+1}} \tilde{b}_{s}^{y^{T}} F_{0} * b_{s}(y) s^{-1} d y d s=\sum_{i=1}^{n} \int_{\mathbf{R}_{+}^{n+1}} \tilde{b}_{\imath, s}^{y^{T}} F_{0} * b_{\imath, s}(y) s^{-1} d y d s,
$$

is a well-defined Fourier multiplier with symbol $\phi$ being a negative, even function homogeneous of degree 0 , which is $2 n-1$ times continuously differentiable on $\mathbf{R}^{n} \backslash\{0\}$.

Proof. First we check that (6.7) is well-defined. Fix $i, 1 \leq i \leq n$. An easy verification shows that the function $\mathbf{R}_{+}^{n+1} \ni(y, s) \mapsto \tilde{b}_{i, s}^{y} \in \mathbf{S}^{\omega}$ is continuous. Let $c=\sup \left\{\left\|\tilde{b}_{j, s}^{\omega, y}\right\|_{\omega}: y \in \mathbf{R}^{n}, s>0,1 \leq\right.$ $j \leq n\}$. For $F \in \mathbf{S}^{\omega}$ we have

$$
\begin{aligned}
\int_{\mathbf{R}_{+}^{n+1}}\left\|\tilde{b}_{\imath, s}^{y}\right\|_{\omega}\left|F_{0} * b_{\imath, s}(y)\right| s^{-1} d y d s & \leq \int_{\mathbf{R}_{+}^{n+1}}\left\|\tilde{b}_{\imath, s}^{\omega, y}\right\|_{\omega}\left|F_{0} * b_{\imath, s}(y)\right| s^{-1} \omega(s) d y d s \\
& \leq c \int_{\mathbf{R}_{+}^{n+1}}\left|F_{0} * b_{\imath, s}(y)\right| s^{-1} \omega(s) d y d s, c<\infty .
\end{aligned}
$$

We claim that there is a constant $C<\infty$, such that for every $i: 1 \leq i \leq n$, the last expression is bounded by $C\|F\|_{\omega}$. Indeed, by Theorem 4.1, we only need to prove that, regarded as a function of $F$, it is bounded on the set of all the Poisson $\omega$-atoms. We use (6.2) to estimate the last integral in case $F=\tilde{G}_{j, r}^{\omega, z}, z \in \mathbf{R}^{n}, r>0,1 \leq j \leq n$ :

$$
\begin{aligned}
\int_{\mathbf{R}_{+}^{n+1}}\left|G_{j, r}^{\omega, z} * b_{\imath, s}(y)\right|^{-1} \omega(s) d y d s & =\omega(r)^{-1} \int_{0}^{\infty}\left\|G_{\jmath} * b_{\imath, s / r}\right\|_{L^{1}\left(\mathbf{R}^{n}\right)} s^{-1} \omega(s) d s \\
& \leq c_{2} \omega(r)^{-1} r \int_{0}^{\infty} \min \left(s^{-2}, r^{-2}\right) \omega(s) d s \leq c^{\prime}
\end{aligned}
$$

where $c^{\prime}<\infty$ is a constant. This gives the result. We see that (6.7) is well-defined and, moreover, that $\|\Phi F\|_{\omega} \leq n C\|F\|_{\omega}$, so the operator $\Phi$ is continuous. Our next task is to show that $\Phi$ 
is a Fourier multiplier with a symbol $\phi$. Clearly, it suffices to check this for the Poisson $\omega$-atoms. Assume that $F$ is such an atom and let $f$ be its boundary value function. Let $t>0$ and $\xi \in \mathbf{R}^{n} \backslash\{0\}$ be fixed. Proceeding as in the proof of Theorem 4.1, we get $I=\widehat{\nabla_{x} \Phi} F_{t}(\xi)=\widehat{\nabla_{x} F_{t}}(\xi) \phi(\xi)$, where

$$
\phi(\xi)=\int_{0}^{\infty} \hat{b}^{T}(s \xi) \hat{b}(s \xi) s^{-1} d s
$$

The change of variable $u=\|\xi\| s$ yields $\phi(\xi)=\int_{0}^{\infty} \hat{b}^{T}(u \xi /\|\xi\|) \hat{b}(u \xi /\|\xi\|) u^{-1} d u$ hence $\phi$ is homogeneous of degree 0 . By analogy to $(6.6)$, we have $\hat{b}_{\imath}(\xi)=i\left(1-\cos \xi_{2}\right) / \xi_{2} \prod_{\jmath=1, \jmath \neq \imath}^{n} \sin \xi_{\jmath} / \xi_{\jmath}, 1 \leq i \leq n$, thus the integrand in $(6.8)$ is equal to

$$
\hat{b}^{T}(s \xi) \hat{b}(s \xi) s^{-1}=-s^{-2 n-1} \prod_{\jmath=1}^{n} \xi_{\jmath}^{-2}\left\{\sum_{t=1}^{n}\left([1-\cos (s \xi)]^{2} \prod_{\jmath=1}^{n} \sin ^{2}\left(s \xi_{\jmath}\right)\right)\right\}
$$

Differentiate this with respect to $\xi_{i}, 1 \leq i \leq n$, at $\xi \neq 0$. The result is of the form $s^{-2 n} W(s \xi)$, where $W$ is certain trigonometric polymomial with smooth coefficients depending only on $\xi$. In consequence, $\phi$ is continuously differentiable on $\mathbf{R}^{n} \backslash\{0\}$. Similar arguments apply to all the derivatives up to the $(2 n-1)$-th order. Finally, note that the integrand in (6.8) is non-positive for every $s>0$, and is negative if $\xi \neq 0$ and $s$ is sufficiently small. Therefore $\phi$ is negative for every $\xi \neq 0$.

Since the integrand is an even function of $\xi$, the same is true for $\phi . \square$

Let $\omega$ be admissible. For $\phi$ as above put $m(\xi)=\phi^{-1}(\xi), \xi \in \mathbf{R}^{n}$. (Recall that $\phi \neq 0$.)

DEFINITION 6.4. Denote by $\mathcal{M}$ the Fourier multiplier $\mathbf{S}^{\omega} \rightarrow \mathbf{S}^{\omega}$ given by the function $m$.

By the above lemma and Theorem $5.1, \mathcal{M}$ is well-defined and continuous.

Now, we are in a position to state and prove our main result.

THEOREM 6.1. Assume that $\omega$ is admissible. For every $F \in \mathbf{S}^{\omega}$ the following equality holds

$$
F=\int_{\mathbf{R}_{+}^{n+1}} \tilde{b}_{s}^{y^{T}} \mathcal{M} F_{0} * b_{s}(y) s^{-1} d y d s \quad \text { (Calderón formula). }
$$

The spaces $\mathbf{S}^{\omega}$ and $\mathbf{B}^{\omega}$ are equal and their norms are equivalent.

Proof. By Lemma 6.2 the integral in (6.9) is well-defined. Since, by definition $\mathcal{M}=\Phi^{-1}$, it is evident that right hand side of (6.9) is the identity of $\mathbf{S}^{\omega}$ and so (6.9) holds. We clearly have

$$
\begin{aligned}
\int_{\mathbf{R}_{+}^{n+1}} \tilde{b}_{s}^{y^{T}} \mathcal{M} F_{0} * b_{s}(y) d y d s & =\sum_{i=1}^{n} \int_{\mathbf{R}_{+}^{n+1}} \tilde{b}_{\imath, s}^{y} \mathcal{M} F_{0} * b_{\imath, s}(y) s^{-1} d y d s \\
& =\sum_{i=1}^{n} \int_{\mathbf{R}_{+}^{n+1}} \tilde{b}_{\imath, s}^{\omega, y} \mathcal{M} F_{0} * b_{\imath, s}(y) s^{-1} \omega(s) d y d s
\end{aligned}
$$

The terms in the last expression are understood as Bochner integrals with respect to the measures of the densities $\mathcal{M} F_{0} * b_{\imath, s}(y) s^{-1} \omega(s), i=1, \ldots, n$. Each term may be approximated in the $\mathbf{S}^{\omega}$ norm by integrals of simple functions all of whose non-zero values are special $\omega$-atoms. Such integrals are in $\mathbf{B}^{\omega}$ and their atomic norm is not greater than $\int_{\mathbf{R}_{+}^{n+1}}\left|\mathcal{M} F_{0} * b_{\imath, s}(y)\right| s^{-1} \omega(s) d y d s \leq C \|$ $\mathcal{M} F\left\|_{\omega} \leq C\right\| \mathcal{M}\|\|_{\omega} i=1, \ldots, n$, with $C$ the same as in the proof of Lemma 6.2 and the operator norm $\|\mathcal{M}\|$ being finite. We see that the conditions in Corollary 2.1 are fulfilled with $N=(C\|\mathcal{M}\| \sqrt{n})^{-1}$ and $M=\sup \left\{\left\|\tilde{b}_{i, s}^{\omega, y}\right\|_{\omega}: y \in \mathbf{R}^{n}, s>0, i=1, \ldots, n\right\}$. The conclusion reads $\mathbf{S}^{\omega}=\mathbf{B}^{\omega}$ and $N\|\cdot\|_{\mathbf{B}^{\omega}} \leq\|\cdot\|_{\omega} \leq M\|\cdot\|_{\mathbf{B}^{\omega}}$. 
REMARK 6.1. Assume that $\omega$ is admissible. Let $\mathcal{F}$ be a finite family of real functions on $\mathbf{R}^{n}$ such that for every $f \in \mathcal{F}$

$$
\begin{gathered}
\int_{\mathbf{R}^{n}}|f(x)|(1+\|x\|) d x<\infty, \int_{\mathbf{R}^{n}} f(x) d x=0, \\
\text { the map } \mathbf{R}^{n} \ni y \mapsto f^{y} \in L^{1}\left(\mathbf{R}^{n}\right) \text { is Lipschitz continuous, } \\
m(\xi)=\left(\sum_{f \in \mathcal{F}} \int_{0}^{\infty} \hat{f}^{2}(\xi t) t^{-1} d t\right)^{-1} \text { satisfies the conditions of Theorem 5.1. }
\end{gathered}
$$

Then $\mathbf{S}^{\omega}$ may be decomposed into the Poisson integrals of the weighted dilations of the functions in $\mathcal{F}$. The proof is the same as in the case of the family $\left\{b_{\imath}: 1 \leq i \leq n\right\}$ considered above. If for example $f \neq 0$ is a real valued, radial, continuously differentiable and compactly supported function such that $\int_{\mathbf{R}^{n}} f(x) d x=0$, then $\mathcal{F}=\{f\}$ satisfies all the above conditions.

The condition (6.12) may be somewhat relaxed since we can "convolve" (see Definition 6.3) $F_{0}$ with both $f_{s}$ and $(\check{f})_{s}, f \in \mathcal{F}$, several times (we write $\check{f}(x)=f(-x)$ ). It follows that we can replace the symbol $\hat{f}^{2}(\xi t)$ in (6.12) by $|\hat{f}(\xi t)|^{2}$ or even by $|\hat{f}(\xi t)|^{2 k}$ with $k \in \mathbf{N}_{+}$.

\section{FURTHER RESULTS}

Let $k \in \mathbf{N}$. Fix a weight function $\omega$ and the corresponding space $\mathbf{S}^{\omega}$. Consider the function family of all the derivatives of the $k$-th order of the function $P_{1}$. If $\omega$ satisfies the condition

$$
\sup _{s>0} \omega(s)^{-1} s^{k} \int_{0}^{\infty}(t+s)^{-(k+1)} \omega(t) d t<\infty,
$$

then the atomic space spanned by the Poisson integrals of the weighted dilations of those functions is identical with $\mathbf{S}^{\omega}$. The condition (7.1) enlarges the set of admissible weights if $k>1$. It should be noticed that if (7.1) holds, the space $\mathbf{S}^{\omega}$ may be decomposed into different classes of atoms related to the Poisson kernel. For example, if $\omega(t)=t^{\alpha}, t>0, \alpha \in(-1,0)$, then $\mathbf{S}^{\omega}$ admits decomposition into Poisson integrals of the weighted dilations of the function $P_{1}$ as well as into those given by derivatives of any fixed order of $P_{1}$.

The result may be generalised. Let a finite function family $\mathcal{F}$ satisfy the conditions (6.11), (6.12) in Remark 6.1. Also, let $\int_{\mathbf{R}^{n}}|f(x)|\left(1+\|x\|^{k}\right) d x<\infty$, and $\int_{\mathbf{R}^{n}} f(x) x^{\alpha} d x=0$, for every multiindex $\alpha$ such that $|\alpha|<k$. Then $\mathbf{S}^{\omega}$ is equal to the atomic space spanned by the Poisson integrals of the weighted dilations of functions $f \in \mathcal{F}$.

Spaces analogous to $\mathbf{S}^{\omega}$ may be defined in terms of derivatives of any fixed order $d \in \mathbf{N}$. Rather than consider the general case, we shall discuss an example. Let a weight function $\omega$ fulfill (7.1) with $k=0$. Define $\mathbf{T}^{\omega}$ as the space of all the functions $U$ harmonic in $\mathbf{R}_{+}^{n+1}$, which satisfy

$$
\|U\|_{\mathbf{T}^{\omega}}=\int_{\mathbf{R}_{+}^{n+1}}|U(x, t)| \omega(t) d x d t<\infty .
$$

(So $d=0$ here.) Since $\left\|s^{-1} \tilde{G}_{\imath, s}^{\omega, y}\right\|_{\mathbf{T}^{\omega}}=\omega(s)^{-1} \int_{0}^{\infty}(t+s)^{-1} \omega(t) d t\left\|G_{1}\right\|_{L^{1}\left(\mathbf{R}^{n}\right)}, y \in \mathbf{R}^{n}, s>$ $0,1 \leq i \leq n$, the set of the functions $s^{-1} \tilde{G}_{\imath, s}^{\omega, y}$ is a bounded subset of $\mathbf{T}^{\omega}$. Denote by $\mathbf{G T}^{\omega}$, the atomic space spanned by these functions. For $i: 1 \leq i \leq n$, and $U \in \mathbf{T}^{\omega}$, define $I_{\imath}(U)=$ $\int_{\mathbf{R}_{+}^{n+1}} s^{-1} \tilde{G}_{\imath, s}^{\omega, y} U(y, s) \omega(s) d y d s$. It is not difficult to check that for every $i: 1 \leq \imath \leq n$, the map $I_{\imath}$ 
is linear and continuous, with values in $\mathbf{S}^{\omega}$, and that $\left(I_{\imath}(\widehat{U})\right)_{t}(\xi)=\frac{1}{2} i \xi_{\Downarrow}\|\xi\|^{-1} \widehat{U}_{t}(\xi), \xi \in \mathbf{R}^{n}, t>0$. Therefore $-4 \sum_{t=1}^{n} I_{t}^{2}$ is the integral representation of the identity of $\mathbf{T}^{\omega}$, and the arguments used before in this paper show that $\mathbf{T}^{\omega}=\mathbf{G T}^{\omega}$. The atomic decomposition of $\mathbf{T}^{\omega}$ is also a consequence of the isomorphism between $\mathbf{S}^{\omega}$ and $\mathbf{T}^{\omega}$. We shall sketch a proof of this isomorphism. Consider the Poisson integrals $\tilde{P}_{s}^{\omega, y}$ of the functions $P_{s}^{\omega, y}, y \in \mathbf{R}^{n}, s>0$. They form a bounded set in $\mathbf{S}^{\omega}$. We claim that the following linear operators are continuous:

$$
\begin{gathered}
\mathbf{T}^{\omega} \ni U \mapsto J(U)=\int_{\mathbf{R}_{+}^{n+1}} \tilde{P}_{s}^{\omega, y} U(y, s) \omega(s) d y d s \in \mathbf{S}^{\omega} \\
\mathbf{S}^{\omega} \ni F \mapsto K_{\imath}(F)=\int_{\mathbf{R}_{+}^{n+1}} s^{-1} \tilde{G}_{\imath, s}^{\omega, y} \partial_{\imath} F(y, s) \omega(s) d y d s \in \mathbf{T}^{\omega}, 1 \leq \imath \leq n .
\end{gathered}
$$

For $U \in \mathbf{T}^{\omega}$, let $V=-4 \sum_{z=1}^{n} K_{\imath} J(U)$. A method similar to that of the proof of Theorem 4.1, gives $\widehat{V}_{t}(\xi)=\widehat{U}_{t}(\xi)$ for every $\xi \in \mathbf{R}^{n}$ and $t>0$, hence $V=U$ in $\mathbf{T}^{\omega}$, and $-4 \sum_{t=1}^{n} K_{t} J$ is the identity of $\mathbf{T}^{\omega}$. We check also that $-4 J \sum_{\imath=1}^{n} K_{\imath}$ is the identity of $\mathbf{S}^{\omega}$, and so the spaces $\mathbf{S}^{\omega}$ and $\mathbf{T}^{\omega}$ are isomorphic. In fact, the spaces are isometric if we endow $\mathbf{S}^{\omega}$ with the norm $\|\cdot\|_{\omega}^{\prime}$ (see Remark 4.2). The corresponding isometry is the operator $2 \sum_{t=1}^{n} K_{2}$, which proves to be the map $\mathbf{S}^{\omega} \ni F \mapsto \partial_{t} F \in \mathbf{T}^{\omega}$. We omit the details.

ACKNOWLEDGEMENT. The author would like to express his gratitude to Dr. Krzysztof Samotij ( Technical University of Wroctaw ) for his inspiration and guidance.

\section{REFERENCES}

1. BLOOM, S. and DESOUZA, G.S. Atomic Decomposition of Generalized Lipshitz Spaces, Illinois J. Math. 33 (1989).

2. DESOUZA, G.S. and SAMPSON, G. A Real Characterisation of the Pre-dual of Bloch Functions, J. Lond. Math. Soc. 27 (2) (1983), 267-276.

3. DESOUZA, G.S. The Atomic Decomposition of Besov-Bergman-Lipshitz Spaces, Proc. Amer. Math. Soc. 94 (1985), 682-686.

4. BONSALL, F.F. Decomposition of Functions as Sums of Elementary Functions, Quart. J. Math. Oxford 37 (2) (1986), 129-136.

5. YOSIDA, K. Functional Analysis, Springer-Verlag, Heildelberg, 1971.

6. FRAZIER, M. and JAWERTH, B. A Discrete Transform and Decompositions of Distribution Spaces, J. Funct. Analysis 93 (1), 1990, 34-170.

7. FRAZIER, M., JAWERTH, B. and WEISS, G., preprint based on lectures given at the conference in Auburn University in 1989.

8. FRAZIER, M. and JAWERTH, B. Decomposition of Besov Spaces, Ind. Univ. Math. J. 34 1985, 777-799.

9. FEICHTINGER, H.G. and GRÖCHENIG, K., Banach spaces related to integrable group representations and their atomic decomposition, I, J. Funct. Analysis 86, 1989, 129-148. 


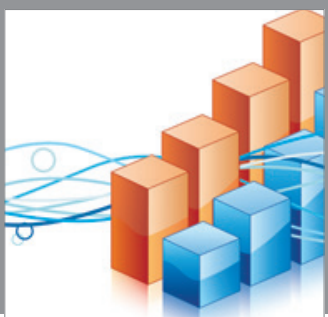

Advances in

Operations Research

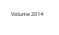

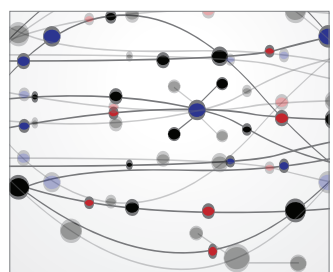

\section{The Scientific} World Journal
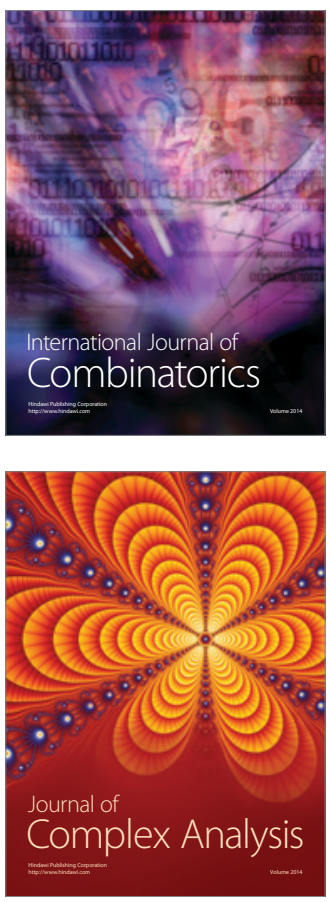

International Journal of

Mathematics and

Mathematical

Sciences
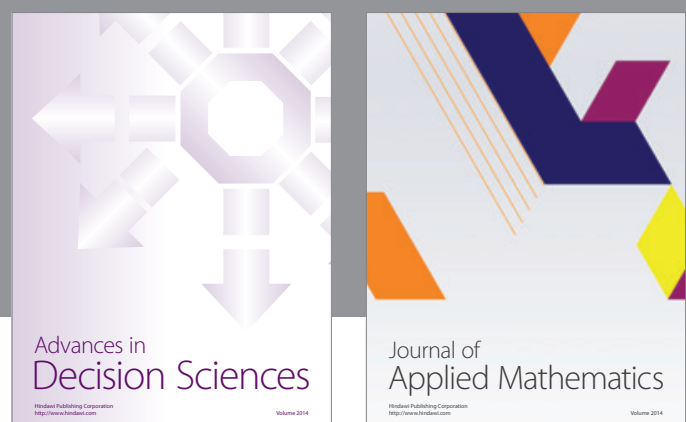

Journal of

Applied Mathematics
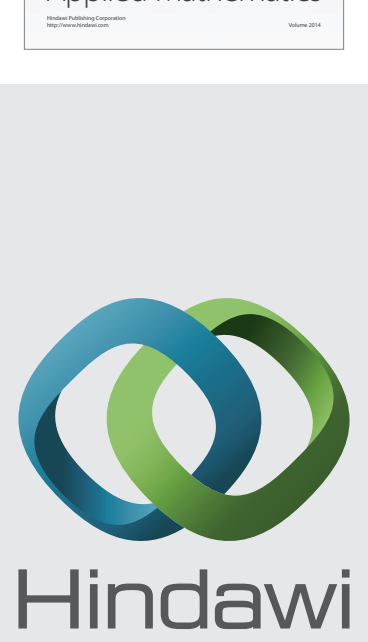

Submit your manuscripts at http://www.hindawi.com
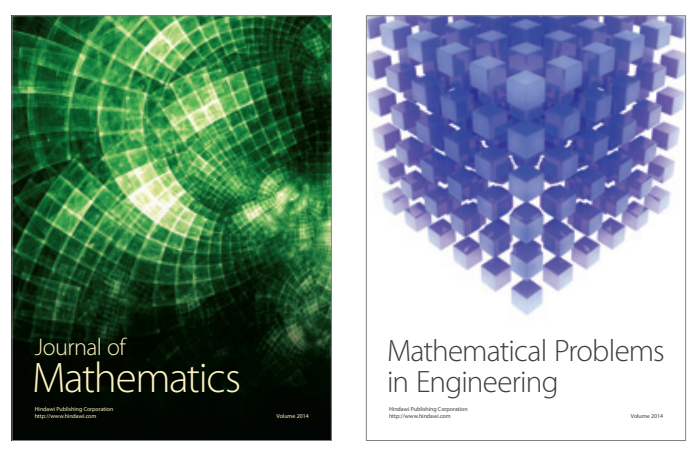

Mathematical Problems in Engineering
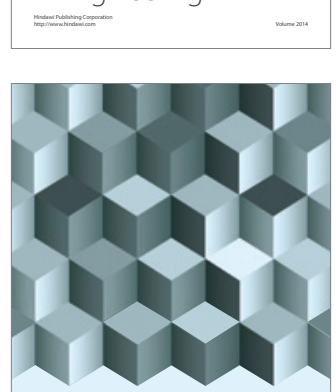

Journal of

Function Spaces
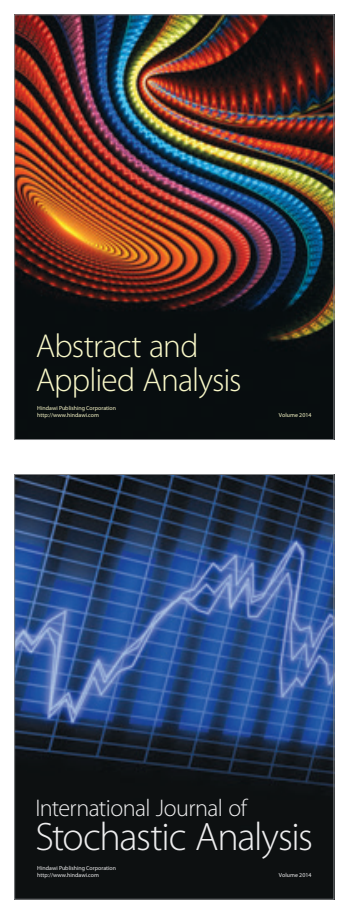

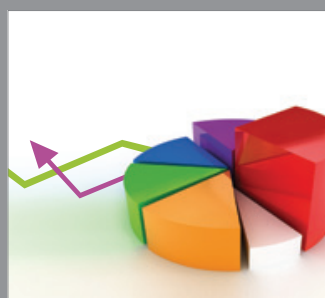

ournal of

Probability and Statistics

Promensencen
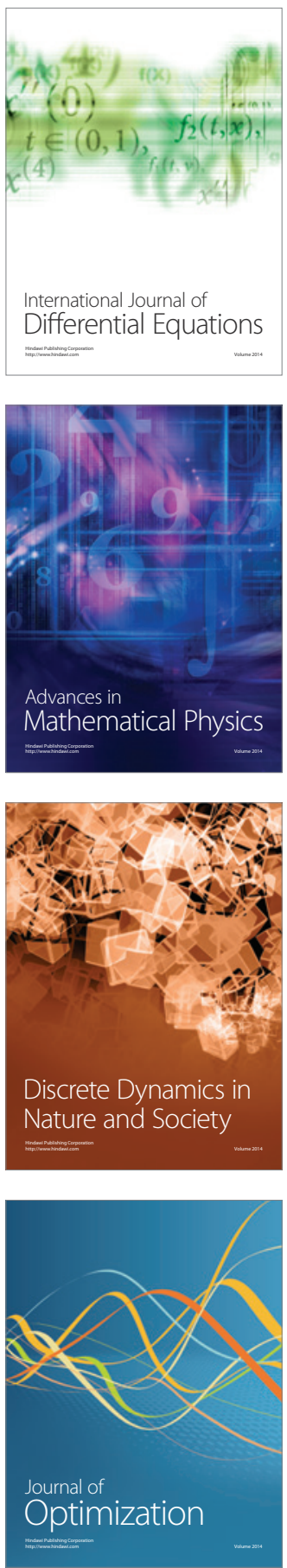